\title{
On the Convergence Rate for a Kernel Estimate of the Regression Function
}

\author{
Mounir ARFI ${ }^{1}$ \\ ${ }^{1}$ University of Sharjah, College of Sciences, Department of Mathematics, Sharjah, United Arab Emirates \\ Correspondence: Mounir ARFI, University of Sharjah, College of Sciences, Department of Mathematics, P.O. Box 27272 \\ Sharjah, United Arab Emirates. E-mail: marfi@sharjah.ac.ae / mounir.arfi@gmail.com
}

Received: December 6, 2015 Accepted: December 28, 2015 Online Published: February 10, 2016

doi:10.5539/ijsp.v5n2p29 URL: http://dx.doi.org/10.5539/ijsp.v5n2p29

\begin{abstract}
We give the rate of the uniform convergence for the kernel estimate of the regression function over a sequence of compact sets which increases to $\mathbb{R}^{d}$ when $n$ approaches the infinity and when the observed process is $\varphi$-mixing. The used estimator for the regression function is the kernel estimator proposed by Nadaraya, Watson (1964).
\end{abstract}

Keywords: Kernel estimate, $\varphi$-Mixing, regression

2000 Mathematics Subject Classifications: 62G05, 62G08.

\section{Introduction}

Let $\left(X_{t}, Y_{t}\right)_{t} \in \mathbb{N}$ be a strictly stationary process where $\left(X_{t}, Y_{t}\right)$ takes on values in $\mathbb{R}^{d} \mathrm{x} \mathbb{R}$ and distributed as $(X, Y)$. Suppose that a segment of data $\left(X_{t}, Y_{t}\right)_{t=1}^{n}$ has been observed.

We are interested in the study of the rate of convergence for a kernel estimate of the regression function, known as:

$$
r(x)=E\left(Y_{t} \mid X_{t}=x\right) \quad t \in \mathbb{N} .
$$

A natural estimator of the function $r($.$) is given by$

$$
r_{n}(x)=\frac{\sum_{t=1}^{n} Y_{t} K\left(\frac{x-X_{t}}{h_{n}}\right)}{\sum_{t=1}^{n} K\left(\frac{x-X_{t}}{h_{n}}\right)} \quad \forall x \in E
$$

Where $E$ stands for the subset $\{x \in \mathbb{R}, f(x)>0\}, f$ being the density of the process $\left(X_{t}\right)$ and $\left(h_{n}\right)$ is a positive sequence of real numbers such that $h_{n} \rightarrow 0$ and $n h_{n}^{d} \rightarrow \infty$ when $n \rightarrow \infty$.

$K$ is a Parzen-Rosenblatt kernel type in the sense of a bounded function satisfying

$$
\int_{\mathbb{R}} K(x) d x=1 \quad \text { and } \quad \lim _{\|x\| \rightarrow \infty}\|x\| K(x)=0
$$

Moreover, it is assumed to be strictly positive and with bounded variation.

The estimation of the regression function has been subject to several investigations, and many authors have been involved. Among others, Devroye (1981), Collomb (1984, 1985), Györfy et al. (1989), Härdle (1990), Bosq (1996), Arfi (1996), Arfi (1997) and Walk (2006).

Watson (1964), for instance, considered the estimation of the conditional expectation as a predictor of $Y$ and applied this method to some climatological time series data; Nadaraya (1964), established the same estimator independently.

Gasser et al. (1984) introduced a kernel estimate for obtaining a nonparametric estimate of a regression function and its derivatives, Sara Van De Ger (1990) proposed an entropy approach to establish rates of convergence for estimators of a regression function and later on Hermann and Ziegler (2004) studied the rates of consistency for a nonparametric estimation of the mode in absence of smoothness assumptions.

Our work is devoted to the rate of the uniform convergence for a kernel estimate of the regression function over an increasing sequence of compact sets under a mixing condition. 


\section{Preliminaries and Assumptions}

We assume that the process $\left(X_{t}\right)_{t \in \mathbb{N}}$ is stationary and $\varphi$-mixing that is

$$
\varphi_{n}=\sup _{A \in \mathcal{M}_{0}^{t}} \sup _{B \in \mathcal{M}_{t+n}^{\infty}}\{|P(B / A)-P(A)|\} \longrightarrow 0, \quad n \rightarrow \infty
$$

where $\mathcal{M}_{0}^{t}$ is the $\sigma$-field generated by $\left\{X_{0}, X_{1}, \ldots, X_{t}\right\}$ and $\mathcal{M}_{t+n}^{\infty}$ is the $\sigma$-field generated by $\left\{X_{t+n}, X_{t+1+n}, \ldots\right\}$

We will make use of the following assumptions

A1. $\exists \Gamma<\infty, \quad \forall x \in \mathbb{R}^{d}, \quad f(x) \leq \Gamma$

and

$\exists \gamma_{n}>0, \quad \forall x \in C_{n}, \quad f(x) \geq \gamma_{n}$

where $C_{n}$ is a sequence of compact sets such that $C_{n}=\left\{x:\|x\| \leq c_{n}\right\}$ and $c_{n} \rightarrow \infty$

A2. $\exists \beta \geq 2, \quad \exists M<\infty$ such that $E\left(|Y|^{\beta}\right) \leq M$

A3. $\exists V<\infty, \quad \forall x \in \mathbb{R}^{d}, \quad E\left[(Y-r(x))^{2} \mid X=x\right] \leq V$

A4. The density $f$ is twice differentiable and its second derivatives are bounded on $\mathbb{R}^{d}$

A5. The kernel $K$ is Lipschitz of ratio $L_{k}$ that is $|K(x)-K(y)| \leq L_{k}\|x-y\|^{\gamma_{1}}$

\section{Main Result}

\section{Theorem}

Assuming that the assumptions A1 through A5 hold, we further assume that the function $r$ is Lipschitz, bounded on $\mathbb{R}^{d}$ and that the bandwith sequence $\left(h_{n}\right)$ satisfies with $y_{n}$ :

$$
\begin{gathered}
n^{\delta} \gamma_{n}^{-1} h_{n}^{-d} y_{n}^{-\beta} \longrightarrow \infty \quad n \rightarrow \infty \\
\forall \epsilon_{0}>0 \quad \sum_{n}\left\{\frac{c_{n}^{d} y_{n}^{d / \gamma_{1}}(\log n)^{d / \gamma_{1}}}{h_{n}^{d\left(1+d / \gamma_{1}\right)}}\right\} \exp \left(-\epsilon_{0}^{2} \frac{n^{1-2 \delta} \gamma_{n}^{2} h_{n}^{d}}{m_{n} y_{n}}\right)<\infty
\end{gathered}
$$

Where $\delta \in] 0,1 / 2\left[, m_{n}\right.$ and $y_{n}$ are two sequences such that:

$$
1 \leq m_{n} \leq n / 2 \text { and } 1 \leq y_{n} \leq \sqrt{n / 2}
$$

If the kernel $K$ is even with $\int z^{2} K(z) d z<\infty$ for $z=\left(z_{1}, \ldots, z_{d}\right)$ and if there exists a constant $D$ such that $\gamma_{n}^{-1} y_{n} n^{\delta} h_{n}^{d}<D$ then if $r$ is continuous, Lipschitz and bounded on $\mathbb{R}$ we have:

$$
n^{\delta} \sup _{\|x\| \leq c_{n}}\left|r_{n}(x)-r(x)\right|=O(1) \quad \text { a.s. } \quad n \rightarrow \infty .
$$

\section{Preliminary Results}

For practical reason, we make the following decomposition:

$$
r_{n}(x)-r(x)=\frac{1}{f(x)}\left\{\left[g_{n}(x)-r(x) f(x)\right]-r_{n}(x)\left[f_{n}(x)-f(x)\right]\right\}
$$

where $\quad g_{n}(x)=\frac{1}{n h_{n}^{d}} \sum_{t=1}^{n} Y_{t} K\left(\frac{x-X_{t}}{h_{n}}\right)$

and $f_{n}(x)=\frac{1}{n h_{n}^{d}} \sum_{t=1}^{n} K\left(\frac{x-X_{t}}{h_{n}}\right)$

This leads to

$$
\sup _{x \in C_{n}}\left|r_{n}(x)-r(x)\right|=\frac{1}{f(x)}\left\{\sup _{x \in C_{n}}\left|g_{n}(x)-r(x) f(x)\right|+\sup _{x \in C_{n}}\left|r_{n}(x)\right|\left|f_{n}(x)-f(x)\right|\right\}
$$

Then if

$$
\sup _{x \in C_{n}}\left|r_{n}(x)\right| \leq y_{n} \quad \text { a.s. } \quad \text { we obtain }
$$




$$
\sup _{x \in C_{n}}\left|r_{n}(x)-r(x)\right|=\gamma_{n}^{-1}\left\{\sup _{x \in C_{n}}\left|g_{n}(x)-r(x) f(x)\right|+y_{n} \sup _{x \in C_{n}}\left|f_{n}(x)-f(x)\right|\right\}
$$

The following Lemma will be used in our proofs

Lemma (Collomb, 1984)

Let $Z_{t}$ be a real centered and $\varphi$-mixing process such tha:

$$
\left|Z_{t}\right| \leq d_{n} \quad E\left(Z_{t}^{2}\right) \leq D_{n} \quad E\left|Z_{t}\right| \leq \delta_{n}
$$

then, $\forall \epsilon>0, \forall n \in \mathbb{N}^{*}$ we have:

$$
P\left\{\left|\sum_{t=1}^{n} Z_{t}\right|>\epsilon\right\} 2 \exp \left\{-\alpha \epsilon+3 \sqrt{e} n \frac{\varphi_{m}}{m}+6 \alpha^{2} n\left[D_{n}+6 \delta_{n} d_{n} \sum_{j=1}^{m} \varphi_{j}\right]\right\}
$$

\section{Lemma 1}

Under the hypotheses of Theorem we have:

$$
\gamma_{n}^{-1} n^{\delta} \sup _{x \in C_{n}}\left|g_{n}(x)-E g_{n}(x)\right| \rightarrow 0 \quad \text { a.s. } \quad n \rightarrow \infty .
$$

Proof:

Because of the possible large values for $Y_{t}$, we use a truncation technique which consists in decomposing $g_{n}$ in $g_{n}^{+}$and $g_{n}^{-}$ where

$$
g_{n}^{+}(x)=\frac{1}{n h_{n}^{d}} \sum_{t=1}^{n} Y_{t} \mathbb{I}_{\left[\left|Y_{t}\right|>y_{n}\right]} K\left(\frac{x-X_{t}}{h_{n}}\right)
$$

and $g_{n}^{-}(x)=g_{n}(x)-g_{n}^{+}(x)$, where $y_{n}$ is the unbounded sequence defined in the Theorem.

We start by showing that:

$$
\gamma_{n}^{-1} n^{\delta} \sup _{\|x\| \leq c_{n}}\left|g_{n}^{-}(x)-E g_{n}^{-}(x)\right| \rightarrow 0 \quad \text { a.s. } \quad n \rightarrow \infty .
$$

To this end, we write:

$$
\begin{gathered}
g_{n}^{-}(x)-E g_{n}^{-}(x)=\sum_{t=1}^{n} \varphi_{t} \quad \text { with } \\
\varphi_{t}=\frac{1}{n h_{n}^{d}}\left\{Y_{t} \mathbb{I}_{\left[\left|Y_{t}\right| \leq y_{n}\right]} K\left(\frac{x-X_{t}}{h_{n}}\right)-E\left[Y_{t} \mathbb{I}_{\left[\left|Y_{t}\right| \leq y_{n}\right]} K\left(\frac{x-X_{t}}{h_{n}}\right)\right]\right\}
\end{gathered}
$$

therefore $E\left(\varphi_{t}\right)=0$;

$\left|\varphi_{t}\right| \leq \frac{2 K_{1} y_{n}}{n h_{n}^{d}}=d_{n}$ where $K_{1}$ is an upperbound of $K$, which permits to write:

$$
E\left|\varphi_{t}\right| \leq \frac{2 \Gamma}{n} E\left|\frac{Y_{t}}{h_{n}^{d}} \mathbb{L}_{\left[Y_{t} \mid \leq y_{n}\right]} K\left(\frac{x-X_{t}}{h_{n}}\right)\right| \leq \frac{2 \Gamma}{n} \int \frac{E\left(\left|Y_{t}\right| / X_{t}=u\right)}{h_{n}^{d}} K\left(\frac{x-u}{h_{n}}\right) d u
$$

Leading by Schwartz inequality and the assumption A3 to:

$$
E\left|\varphi_{t}\right| \leq \frac{2 \Gamma}{n} \int \frac{\left(r^{2}(u)+V\right)^{1 / 2}}{h_{n}^{d}} K\left(\frac{x-u}{h_{n}}\right) d u \leq \tau_{1} n^{-1}
$$

where $\tau_{1}$ is a positive constant.

Now, same arguments give:

$$
E\left(\varphi_{t}\right)^{2} \leq \frac{2 \Gamma}{n^{2}} \int \frac{\left(r^{2}(u)+V\right)}{h_{n}^{2 d}} K\left(\frac{x-u}{h_{n}}\right) d u \leq v n^{-2} h_{n}^{-d}
$$

where $v$ is a positive constant.

We apply the Collomb inequality with $\alpha=1 /\left(4 m_{n} d_{n}\right)$ and we obtain for all $\epsilon_{n}>0$ :

$$
P\left(\left|g_{n}^{-}(x)-E g_{n}^{-}(x)\right|>\epsilon_{n}\right) \leq C_{1} \exp \left(-C_{2} \epsilon_{n}^{2} \frac{n h_{n}^{d}}{m_{n} y_{n}}\right)
$$


where $C_{1}$ and $C_{2}$ are two positive constants.

Next, we cover $C_{n}$ by $\mu_{n}^{d}$ spheres in the shape of $\left\{x:\left\|x-x_{j n}\right\| \leq c_{n} \mu_{n}^{-1}\right\}$ where $1 \leq j \leq \mu_{n}^{d}$.

And we make the following decomposition:

$$
\left|g_{n}^{-}(x)-E g_{n}^{-}(x)\right| \leq\left|g_{n}^{-}(x)-g_{n}^{-}\left(x_{j n}\right)\right|+\left|g_{n}^{-}\left(x_{j n}\right)-E g_{n}^{-}\left(x_{j n}\right)\right|+\left|E g_{n}^{-}\left(x_{j n}\right)-g_{n}^{-}(x)\right|
$$

and we have

$$
\left|g_{n}^{-}(x)-g_{n}^{-}\left(x_{j n}\right)\right| \leq \frac{y_{n}}{n h_{n}^{d}} \sum_{t=1}^{n}\left|K\left(\frac{x-X_{t}}{h_{n}}\right)-K\left(\frac{x_{j n}-X_{t}}{h_{n}}\right)\right|
$$

The kernel $K$ being Lipschitz we obtain

$$
\begin{gathered}
\left|g_{n}^{-}(x)-g_{n}^{-}\left(x_{j n}\right)\right| \leq L_{K} \frac{y_{n}}{h_{n}^{d+\gamma_{1}}}\left\|x-x_{j n}\right\|^{\gamma_{1}} \\
\left|g_{n}^{-}(x)-g_{n}^{-}\left(x_{j n}\right)\right| \leq L_{K} \frac{y_{n}}{h_{n}^{d+\gamma_{1}}} c_{n}^{\gamma_{1}} \mu_{n}^{-\gamma_{1}} \\
\left|g_{n}^{-}(x)-g_{n}^{-}\left(x_{j n}\right)\right| \leq \frac{1}{\log n}
\end{gathered}
$$

If we choose

$$
\mu_{n}=L_{K}^{1 / \gamma_{1}} \frac{y_{n}^{1 / \gamma_{1}} c_{n}(\log n)^{1 / \gamma_{1}}}{h_{n}^{d / \gamma_{1}+1}} \longrightarrow \infty
$$

Thus we obtain:

$$
\sup _{x \in C_{n}}\left|g_{n}^{-}(x)-E g_{n}^{-}(x)\right| \leq \sup _{1 \leq j \leq \mu_{n}^{d}}\left|g_{n}^{-}\left(x_{j n}\right)-E g_{n}^{-}\left(x_{j n}\right)\right|+\frac{2}{\log n}
$$

Therefore, if we apply $\mu_{n}^{d}$ times the Lemma of Collomb, we obtain

$$
P\left(\sup _{x \in C_{n}}\left|g_{n}^{-}(x)-E g_{n}^{-}(x)\right|>2 \epsilon_{n}\right) \leq C_{1} \mu_{n}^{d} \exp \left(-C_{2} \epsilon_{n}^{2} \frac{n h_{n}^{d}}{m_{n} y_{n}}\right)
$$

Now if we choose $\epsilon_{n}=n^{-\delta} \gamma_{n} \epsilon_{0}$ for a certain $\epsilon_{0}>0$, we obtain accordingly with the hypotheses of the Theorem:

$$
P\left(\gamma_{n}^{-1} n^{\delta} \sup _{\|x\| \leq c_{n}}\left|g_{n}^{-}(x)-E g_{n}^{-}(x)\right|>2 \epsilon_{0}\right) \leq C_{1} L_{K}^{d / \gamma_{1}} \frac{y_{n}^{d / \gamma_{1}} c_{n}^{d}(\log n)^{d / \gamma_{1}}}{h_{n}^{d\left(1+d / \gamma_{1}\right)}} \exp \left(-C_{2} \epsilon_{0}^{2} \frac{n^{1-2 \delta} \gamma_{n}^{2} h_{n}^{d}}{m_{n} y_{n}}\right)
$$

The hypotheses of the Theorem permit to conclude that:

$$
\gamma_{n}^{-1} n^{\delta} \sup _{\|x\| \leq c_{n}}\left|g_{n}^{-}(x)-E g_{n}^{-}(x)\right| \longrightarrow 0, \quad \text { a.s. } \quad n \rightarrow \infty
$$

It remains to show that:

$$
n^{\delta} \gamma_{n}^{-1} \sup _{\|x\| \leq c_{n}}\left|g_{n}^{+}(x)-E g_{n}^{+}(x)\right| \rightarrow 0, \quad \text { a.s. } \quad n \rightarrow \infty .
$$

For practical reason, we write:

$$
n^{\delta} \gamma_{n}^{-1} \sup _{\|x\| \leq c_{n}}\left|g_{n}^{+}(x)-E g_{n}^{+}(x)\right| \leq E_{n}+F_{n}
$$

Where,

$$
E_{n}=\frac{n^{\delta} \gamma_{n}^{-1}}{n h_{n}^{d}} \sup _{\|x\| \leq c_{n}}\left|\sum_{t=1}^{n} Y_{t} \mathbb{I}_{\left(\left|Y_{t}\right|>y_{n}\right)} K\left(\frac{x-X_{t}}{h_{n}}\right)\right|
$$

And we have

$$
\left(E_{n} \neq 0\right) \subset\left\{\exists t_{0} \in[1,2,3, \ldots, n] \text { such that }\left|Y_{t_{0}}\right|>y_{n}\right\}
$$

the above leads to

$$
\left(E_{n} \neq 0\right) \subset \bigcup_{t=1}^{n}\left\{\left|Y_{t}\right|>y_{n}\right\}
$$




$$
\begin{gathered}
P\left(E_{n} \neq 0\right) \leq \sum_{t=1}^{n} P\left(\left|Y_{t}\right|>y_{n}\right)=n P\left(\left|Y_{t}\right|>y_{n}\right) \\
\sum_{n} P\left(E_{n} \neq 0\right) \leq \sum_{n} P\left(\left|Y_{t}\right|>y_{n}\right) \leq \sum_{n} n y_{n}^{-\beta} E|Y|^{\beta} \\
\sum_{n} P\left(E_{n} \neq 0\right) \leq c_{4} \sum_{n} n y_{n}^{-\beta}<\infty
\end{gathered}
$$

where $c_{4}$ is a positive constant.

Then $E_{n} \rightarrow 0$, a.s., $\quad n \rightarrow \infty$ and $\sup \left|Y_{t}\right| \leq y_{n}$ a.s.

The kernel $K$ being strictly positive, we conclude that $\left|r_{n}(x)\right| \leq y_{n} \quad$ a.s.

Moreover,

$$
\begin{gathered}
F_{n}=\frac{n^{\delta}}{\gamma_{n} n h_{n}^{d}} \sup _{\|x\| \leq c_{n}}\left|\sum_{t=1}^{n} E\left[Y_{t} \mathbb{I}_{\left[\left|Y_{t}\right|>y_{n}\right]} K\left(\frac{x-X_{t}}{h_{n}}\right)\right]\right| \\
F_{n} \leq \frac{n^{\delta}}{\gamma_{n} h_{n}^{d}} K_{1} E\left[|Y| \mathbb{I}_{|Y|>y_{n}}\right] \\
F_{n} \leq \frac{n^{\delta}}{\gamma_{n} h_{n}^{d}} K_{1}\left(E\left(Y^{2}\right)\right)^{1 / 2}\left(P\left[|Y|>y_{n}\right]\right)^{1 / 2} \leq c_{5} n^{\delta} \gamma_{n}^{-1} h_{n}^{-d} y_{n}^{-\beta / 2} \rightarrow 0, \quad n \rightarrow \infty
\end{gathered}
$$

where $c_{5}$ is being a positive constant.

\section{Lemma 2}

Under the assumptions of the Theorem, we have:

$$
n^{\delta} \gamma_{n}^{-1} \sup _{x \in \mathbb{R}^{d}}\left|E g_{n}(x)-r(x) f(x)\right| \rightarrow 0, \quad n \rightarrow \infty
$$

Proof:

$$
\begin{gathered}
E g_{n}(x)-r(x) f(x)=\frac{1}{n h_{n}^{d}} E\left\{\sum_{t=1}^{n} Y_{t} K\left(\frac{x-X_{t}}{h_{n}}\right)\right\}-r(x) f(x) \\
E g_{n}(x)-r(x) f(x)=\frac{1}{h_{n}^{d}} \int_{\mathbb{R}^{d}} r(u) K\left(\frac{x-u}{h_{n}}\right) f(u) d u-r(x) f(x)
\end{gathered}
$$

We write $z=(x-u) / h_{n}$ and we obtain

$$
E g_{n}(x)-r(x) f(x)=\int_{\mathbb{R}^{d}}\left[r\left(x-z h_{n}\right)-r(x)\right] K(z) f\left(x-z h_{n}\right) d z+r(x) \int K(z)\left[f\left(x-z h_{n}\right)-f(x)\right] d z
$$

Assuming that the function $r($.$) is Lipschitz of ratio 1$ and order 1 provides

$$
\left|\int_{\mathbb{R}^{d}}\left[r\left(x-z h_{n}\right)-r(x)\right] K(z) f\left(x-z h_{n}\right) d z\right| \leq h_{n} \Gamma \int|Z| K(z) d z
$$

Now a Taylor expansion, the Bochner lemma and the fact that the function $r$ is bounded permit to conclude that

$$
n^{\delta} \gamma_{n}^{-1} \sup _{x \in \mathbb{R}^{d}}\left|E g_{n}(x)-r(x) f(x)\right| \rightarrow 0, \quad n \rightarrow \infty
$$

\section{Lemma 3}

Under the assumptions of the Theorem, we have:

$$
\lim _{n \rightarrow \infty} \frac{y_{n} n^{\delta}}{\gamma_{n}} \sup _{\|x\| \leq c_{n}}\left|f_{n}(x)-E f_{n}(x)\right|=0 \text { a.s. }
$$

Proof:

This is a particular case of Lemma 1 when $Y_{t}=1$ and $\epsilon=\epsilon_{0} \gamma_{n} n^{-\delta} y_{n}^{-1}$ for a certain $\epsilon_{0}>0$.

\section{Lemma 4}


Under the assumptions of the Theorem, we have:

$$
\lim _{n \rightarrow \infty} \frac{y_{n} n^{\delta}}{\gamma_{n}} \sup _{x \in \mathbb{R}^{d}}\left|E f_{n}(x)-f(x)\right|=0
$$

Proof:

We write

$$
E f_{n}(x)-f(x)=\frac{1}{h_{n}^{d}} \int[f(u)-f(x)] K\left(\frac{u-x}{h_{n}}\right) d u
$$

A Taylor expansion and the hypotheses of the Theorem and the Bochner lemma permit to conclude.

\section{Proof of the Theorem}

The lemma 1, Lemma 2, Lemma 3, and Lemma 4 permit to conclude.

\section{Acknowledgments}

The author would like to thank the referees for their comments and criticisms about this paper.

\section{References}

Arfi, M. (1996). Sur la Regression non parametrique d’ un processus stationnaire mélangeant ou ergodique. Doctorate Thesis of University Paris 6.

Arfi, M. (1997). On General Estimate of the Regression Function. Statistics \& Decisions, 15, 191-200. http://dx.doi.org/10.1524/strm.1997.15.2.191

Bosq, D. (1996). Nonparametric statistics for stochastic processes. Lecture Notes in Statistics, 110, Springer Verlag. http://dx.doi.org/10.1007/978-1-4684-0489-0

Collomb, (1984). Proprietes de Convergence Presque Sure du Predicteur a Noyau. Z. Wahrsch. Verw., Gebiete., 66, 441-460. http://dx.doi.org/10.1007/BF00533708

Collomb, G. (1985) Nonparametric regression; an up to date bibliography. Statistics, 16, 309-324. http://dx.doi.org/10.1080/02331888508801860

Devroye, L. (1981). On the Almost Eveywhere Convergence of Nonparametric Regression Function Estimates. The Annals of Statistics, 9, 1310-1319. http://dx.doi.org/10.1214/aos/1176345647

Gasser, T., \& Hans-Georg Müller. (1984). Estimating Regression Functions and Their Derivatives by the Kernel Method. Scand. J. Statist., 11, 171-185.

Györfy, L., Härdle, W., Sarda, P., \& Vieu, P. (1989). Nonparametric Curve Estimation from Time Series. Lecture Notes in Statistics, 60, Springer Verlag.

Härdle, W. (1990). Applied Nonparametric Regression. Cambridge Univ. Press.

Hermann, E., \& Ziegler, K. (2004). Rates of Consistency for a Nonparametric Estimation of the Mode in Absence of Smoothness Assumptions. Statistics \& Probability Letters, 68, 359-368. http://dx.doi.org/10.1016/j.spl.2004.04.005

Nadaraya, E. A. (1964). On estimating regression. Theory of Probability and its Application, 9, 141-142. http://dx.doi.org/10.1137/1109020

Sara Van De Ger. (1990). Estimating a Regression Function. The Annals of Statistics, 18(2), 907-924. http://dx.doi.org/10.1214/aos/1176347632

Walk, H. (2006). Almost sure Convergence Properties of Nadaray-Watson Regression Estimates. International Series in Operations Research \& Management Science, 46, Modeling Uncertainty, 201-223.

Watson, G. S. (1964) Smooth regression analysis. Sankhya, A 26, 359-372.

\section{Copyrights}

Copyright for this article is retained by the author(s), with first publication rights granted to the journal.

This is an open-access article distributed under the terms and conditions of the Creative Commons Attribution license (http://creativecommons.org/licenses/by/3.0/). 